\title{
Using Pen and Paper to Control Networked Appliances
}

\author{
Mario Kolberg and Evan H. Magill \\ Department of Computing Science and Mathematics \\ University of Stirling, Stirling FK9 4LA \\ United Kingdom \\ \{mko,ehm\}@cs.stir.ac.uk
}

User interfaces to control networked household appliances are often inadequate. Either they are too simplistic or they are too complex and complicated relying on a PC based interface. Therefore a user interface that supports complex functionalities and is easy to use is required.

In this paper, digital pen and paper are presented as a suitable interface. Digital pens are only marginally larger than their traditional counterparts, and digital paper is ordinary paper with an almost invisible unique pattern printed on it. Pen and paper are well known to users and thus an important barrier to the acceptance of networked appliances is removed.

The approach is integrated with an OSGi gateway allowing for flexibility with respect to the protocol used to communicate with the appliance. It is also shown how the approach can be applied to other appliances in and beyond the home.

\section{Introduction}

The Smith family is on a short weekend break in the country. Daughter Grace is questioning her parents if the PVR back at home has been programmed to record her favorite TV soap so she could watch it on their return. Unfortunately father and mother Smith had other things on their mind and forgot. However, father Smith rescues the weekend by stepping into a local newsagent and purchasing a TV magazine which features 'tick-to-program' functionality. Using his digital pen, father Smith ticks the boxes next to the programs to be recorded on the PVR at home. After ticking the 'Send' box on the page and a short wait, he receives a text message on his mobile phone confirming that the PVR is now programmed to record the selected TV shows.

This example illustrates two main advantages: firstly, the PVR (Personal Video Recorder) at home can be programmed remotely, and secondly and arguably more importantly, the user interface to the PVR is much more user friendly and intuitive than in the past. Home appliances are targeted at the end consumer. However, a large number of end consumers are put off by complicated user interfaces. For instance, the above scenario could also be realized by using a webpage offered by the PVR. While some consumers will be happy using a web interface from their PCs or PDAs, others who are not familiar with a PC are not used to this kind of interface. Even a touch screen or stylus based input do not fully address the problem. It is still a PC interface using PC style menus and submenus. Consequently, they will either not use many of the advanced features of the appliances, or worse, are discouraged from buying advanced home appliances. The blinking 12:00 on many VCRs is a typical example of this behaviour. Because of the complicated user interface, the VCR is not setup properly, and consequently no timed recordings can be scheduled. Hence the appliance can not be used fully. Furthermore, some consumers just may find it impractical or inconvenient to control their appliances using a laptop or PDA. Also the webpage of a complex appliance, such as a 
PVR, displayed on a PDA or mobile phone will certainly look confusing. Pen and paper are rather non-intrusive and portable.

For some parents the example above may sound too good to be true. However, this is perfectly possible today by integrating different existing technologies, mainly a programmable PVR, an OSGi gateway, and Anoto enabled pen and paper technology [1]. Anoto functionality enabled pens are currently manufactured by Nokia, Logitech, and Maxwell. There are also office type paper products such as diaries and notebooks commercially available. PVRs are readily commercially available as are OSGi framework implementations. Here we show how, with the addition of some OSGi bundles, it is possible to integrate such technologies to provide an intuitive and portable interface.

In the following, the technology behind the above example and the integration are discussed. While so far a PVR has been used as the example, the following discussion can be applied to appliances connected to an OSGi gateway in general. Some such examples are discussed in Section 3.4.

\section{Networked Appliances}

Networked appliances are consumer devices which are networked. As such, an increased number of networked appliances may assume a networked home with an always-on Internet connection. Examples include PVRs, TVs, stereos, fridges, stoves, lights, curtain drawers (puller), and security cameras.

\subsection{Deploying Networked Appliances in the Home}

Networked appliances are typically deployed inside the home and are connected to a Local Area Network (LAN). However, appliances use a large number of different protocols to communicate, such as UPnP (Universal Plug and Play), X-10, Bluetooth, HAVi (Home Audio/Video Interoperability), and MHP (Multimedia Home Platform). Consequently, appliances employing different protocols cannot communicate with each other. This issue can be solved by the use of a Residential Gateway which can act as a "glue" or bridge between protocols [2]. 


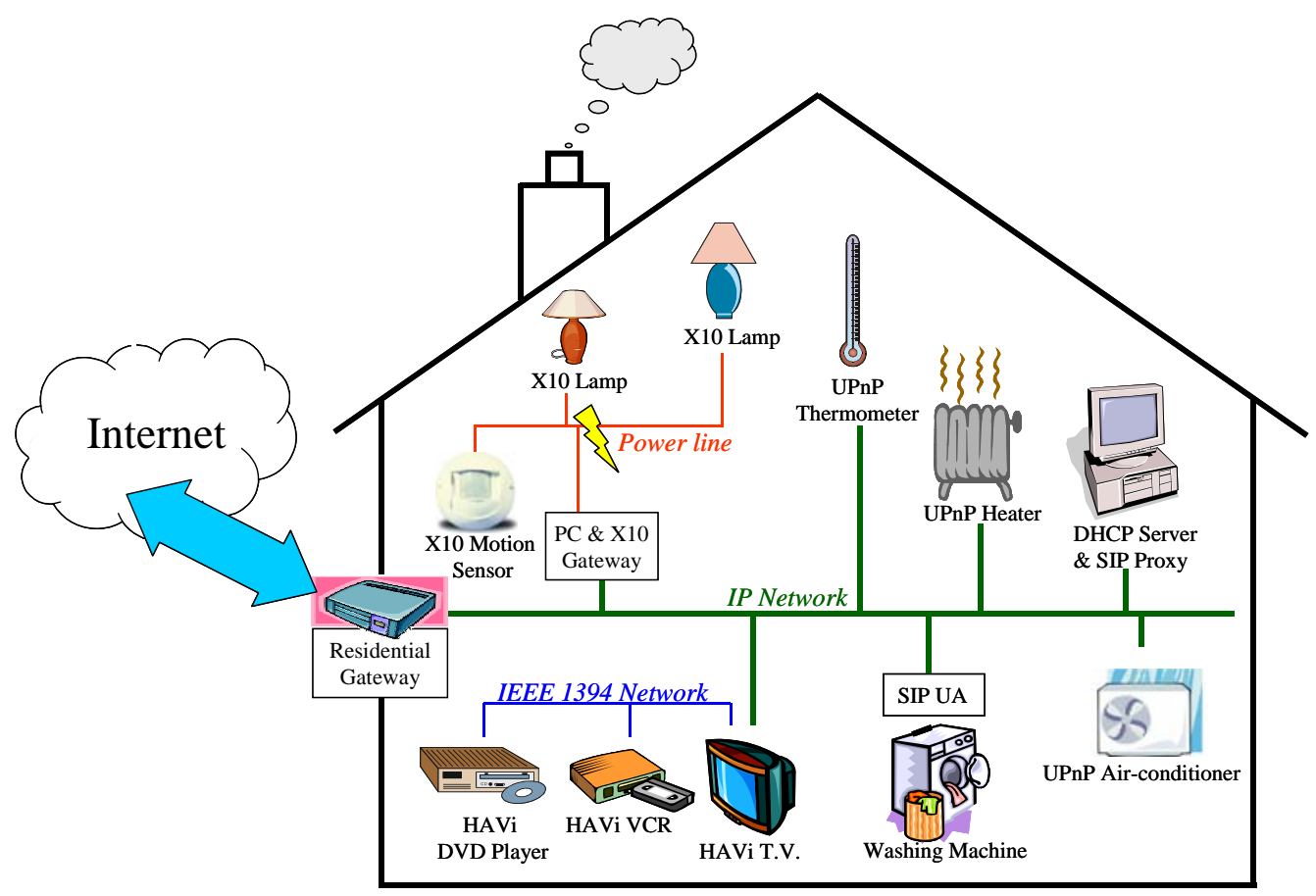

Figure 1: Appliances in the Home.

Furthermore, the Residential Gateway links the home with the Internet and offers services such as Network Address Translator and Firewall functionalities (Figure 1). Importantly, the gateway also hosts the software services which control the appliances. One example of such a gateway is the Open Services Gateway [3,4] standardised by the Open Services Gateway Initiative (OSGi). OSGi aims to deliver a platform for providing services over the lower-level networking standards. OSGi runs on any Java based system, and may be provided in a set-top box, or a standard PC.

\subsection{OSGi}

OSGi offers a set of APIs for managing user services, and provides a means for device access, configuration management and user management. OSGi is neutral with respect to the services it offers. Rather it is concerned with the (remote) management of those services (installing, starting, stopping and updating) without the need to restart the gateway. Security is also a prime concern of the initiative.

OSGi offers an ideal platform for the integration of the different technologies used in this approach. OSGi's inherently built-in ability to integrate a wide range of different network protocols and allowing applications to control appliances within the home without awareness of the underlying communications protocol is a crucial point in this approach. For instance, OSGi can be used to integrate the pen and paper technology with an UPnP A/V PVR, or DLNA PVR, or a HAVI PVR, or any appliance using any other protocol. Clearly, the approach presented in this paper could have been implemented with a particular focus to any one of the protocols, however, that would have restricted the use of the approach with appliances communicating using this single protocol. OSGi offers more flexibility here. Peerto Peer architectures, such as JXTA, may also be applicable. However, at present, they cannot match the strong protocol integration feature of OSGi. Other architectures, such as RUNES 
focus on slightly different domains (sensor networks). Furthermore, it has a wide area focus, whereas the approach (and OSGi) focuses on a single domain (the home). Indeed, some work has been carried out to allow for inter-domain communication with OSGi [5]. ePerSpace which builds on OSGi may have been applicable, but no implementations were readily available.

The OSGi framework is relatively simple, but offers many possibilities for service providers. However, the interest here is in its ability to control devices regardless of their underlying protocol and to allow their remote control. OSGi uses the concept of bundles which represent either device drivers or services. Services offer some particular functionality to other services or the outside world. Device drivers allow the communication with physical devices. There is one device driver for each supported network protocol, such as UPnP or X10.

When a new device joins the network, the driver within the framework adds a representation of it to the framework. The newly added device is now available for use by services on the framework. Its appearance is just like another service, albeit with rather low-level functionality. Thus other services may use it via the uniform OSGi service interface. Consequently, the framework may offer a collection of UPnP, X10 and other devices and (user) services can control devices irrespective of the underlying protocol used by a the device. For instance, an entertainment service may control an UPnP PVR and TV as well as a X10 curtain puller and lamp. Thus OSGi can be seen as the "glue" which links devices (which use different network protocols) together. An example is shown in Figure 2.

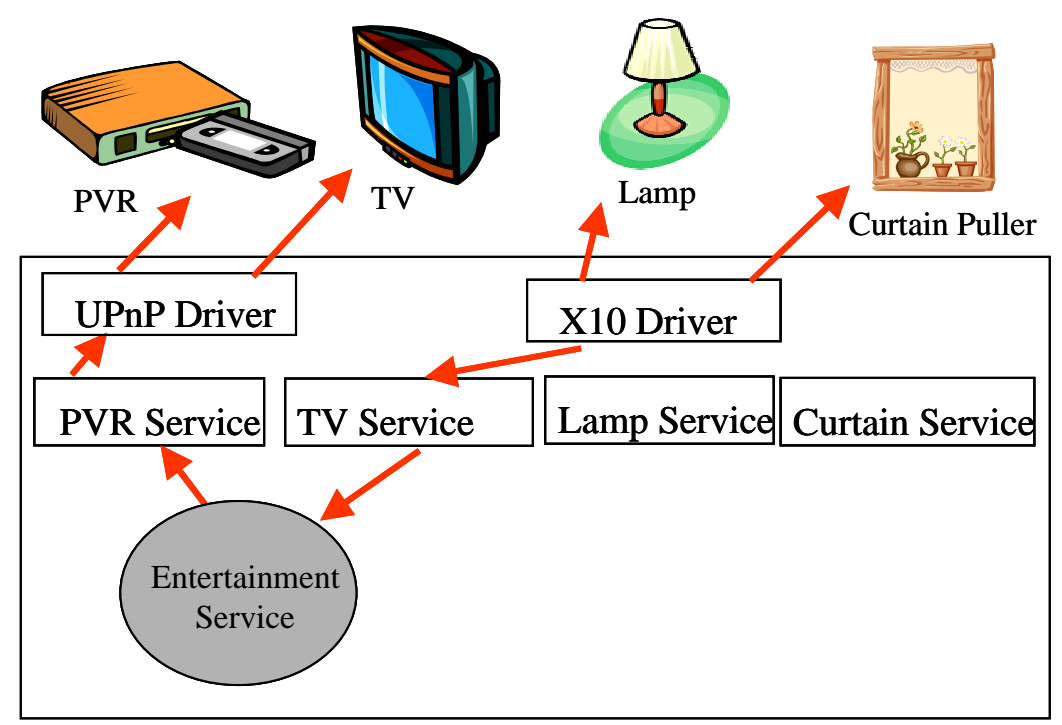

Figure 2: An OSGi Gateway Configuration.

One major obstacle in deploying networked appliances and OSGi is the user interface. OSGi uses a typical computer based interface to configure and remotely control the devices and services. This may be a basic command line interface or a graphical (web) interface. Both interfaces are largely inadequate for computer illiterate users and thus are a major barrier to the mass-market adoption of the technology. The next section discusses how Anoto enabled Pen and Paper can be used to solve this issue. 


\section{Using Pen and Paper to Control Appliances}

\subsection{Digital Pen and Paper}

Digital pens and paper enabled with Anoto functionality are an extremely easy to use interface to computer applications. Anoto enabled paper is standard paper with a special pattern printed on it. The dots are $0.3 \mathrm{~mm}$ apart. These dots are slightly displaced from a grid structure to form the proprietary Anoto pattern. A minute portion of the total pattern uniquely defines its position in the full pattern, 60000000 square kilometers, which is equivalent to an area exceeding that of Europe and Asia combined. The pattern is almost invisible to the human eye and printed using carbon ink. It can be overprinted with any non-carbon ink. Effectively, the pen 'sees' the pattern and the human eye sees the form overlay.

To allow for different applications, a portion of the pattern can be linked to a particular application. This is done by associating a URL with a pattern. All pen strokes written on a page are sent to the URL. At that URL an application in the form of a Java servlet is listening for this information. Thus the information can be processed in an application specific way. For instance, when writing in a diary, the writing information will be associated with the diary application on the computer. Even within a page the writing can be located precisely. If the user ticks a check box or adds an appointment for a particular time in their diary, the linked application can use this information. In other words, the application is aware of where the user has been writing on the page. While the paper with the pattern identifies the application, the pen identifies the user. This way the recorded writing in a diary can be associated with the correct application for the correct user.

\begin{tabular}{|c|c|c|c|}
\hline 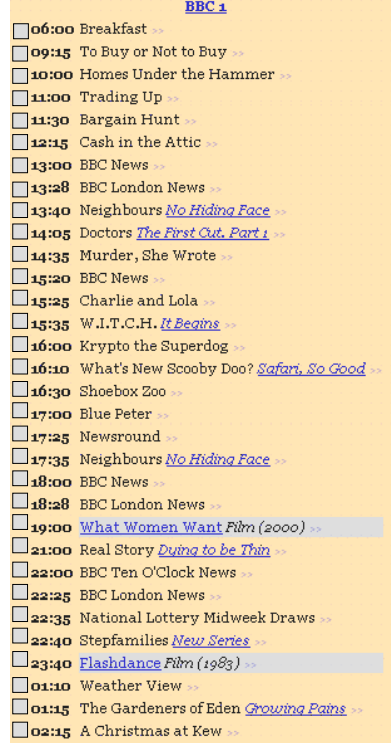 & 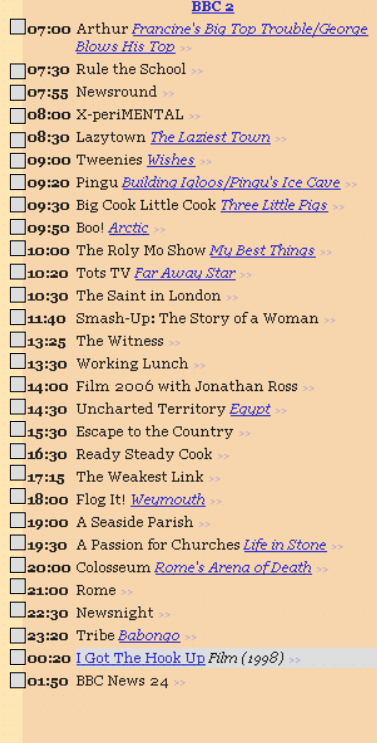 & 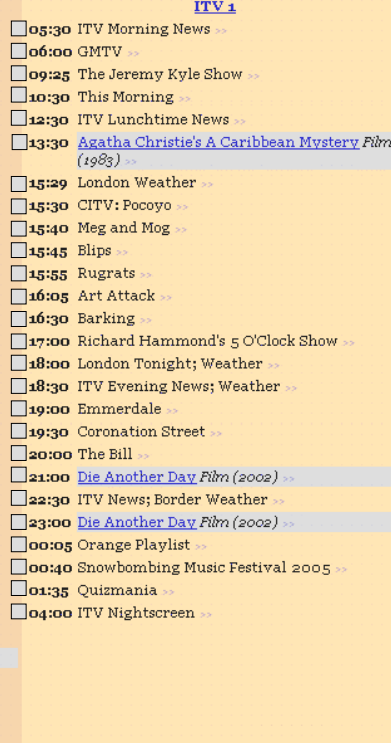 & 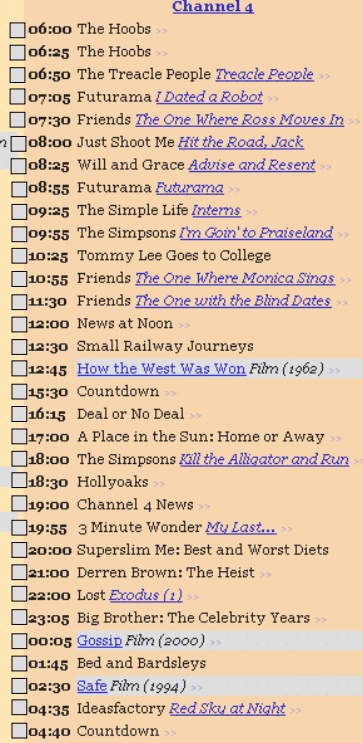 \\
\hline
\end{tabular}

Figure 3: Sample TV listing with Anoto functionality enabled tick boxes (based on bleb.org).

When writing with a digital pen on paper printed with the pattern, digital snapshots of the pattern are taken (about 50 per second). That is, the pen records its movement over the pattern with a tiny camera and stores the information in memory inside the pen. Every snapshot contains enough information to make a calculation of the exact position of the pen. "The intelligence in the paper”, derived from the pattern, makes it possible to perform operations 
by just ticking a box with the pen. Once a page is completed the information is transferred to the mobile phone using Bluetooth, from where it is sent on to the service provider. The service provider processes the information and commonly sends it to its target destination (e.g. the user's home).

This technology offers an ideal interface to control networked appliances, such as a PVR. All that is required is a paper form that reflects the broadcast programs for the next week. An ideal partner would be a TV magazine printing a small column with the Anoto pattern next to the listed programs. For each program there would be a tick box in this column. To program the PVR to record a particular TV show, all the user has to do, is to tick the corresponding box. An example is shown in Figure 3.

\subsection{Integrating the Pen Interface with OSGi}

For this approach to work, a number of steps are required. Firstly, the user selects the desired TV program by ticking the corresponding box in the magazine. As the user does the pen stroke, the pen's camera picks up the pattern of the box and stores the pen stroke in internal memory. Once a paper form is filled in, the data together with the ID of the used pen is sent via Bluetooth to the paired mobile phone handset. There is no special software running on the mobile phone. It only needs to support Bluetooth 1.1 and the Bluetooth profiles DUN (Dialup Networking) and OPP (Object Push Profile). The large majority of current mobile phones fulfill these requirements. The pen mirrors the Bluetooth profiles from the mobile phone.

The mobile phone then acts like a modem and using GPRS forwards the data onto the service provider. The service provider hosts an application (Java servlet) which receives and processes the pen stroke data. This requires that the URL for the pattern used points to the service provider application. This way, the correct service provider is identified, and the pen strokes are sent to the right application.

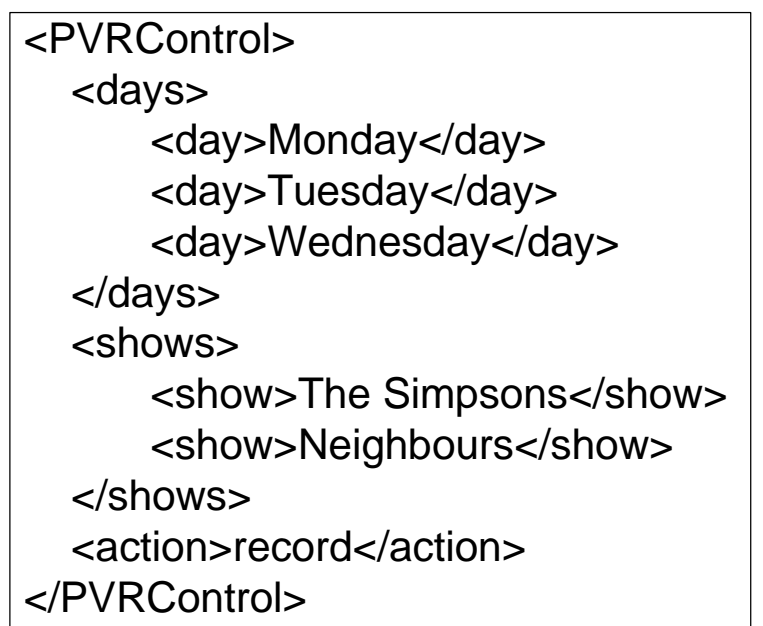

Figure 4: Sample XML data generated from Pen Strokes.

For this example, the data is translated into an XML based paper independent data format. For the application discussed in Section 3.3, this contains simple tags describing the user selections, i.e. the selected days, TV shows, and action. Figure 4 depicts an example. 
The ID of the pen is sent along with the pen stroke data to the service provider application and is there used to identify the particular customer whose PVR should be programmed. The XML data is then sent to a service in the OSGi gateway of the pen owner's home. The pen service then checks the received data against an Electronic Program Guide (EPG). Which EPG database is used is secondary to the approach, and depends of the location of the customer. For instance for a customer in the UK, a UK database needs to be used. For the experiments we have used data from www.bleb.org, which offers program information in XML format. XMLTV is an alternative approach, also using XML to offer the data. Typically, these services offer a 7 day EPG. So the EPG OSGi service checks the database at least every seven days and stores the received data locally. From the EPG, the pen service finds screenings of the chosen TV shows on the selected days. It extracts all required information needed to program the PVR. This includes, channel number, screening dates, start time and end time.

Finally the program request is sent to the PVR. As is common in OSGi, this request is initially directed to the service representing the PVR (a reference to this is obtained from the OSGi registry), then forwarded onto the driver bundle of the protocol used by the PVR, and then sent to the physical PVR. Figure 5 shows this process.

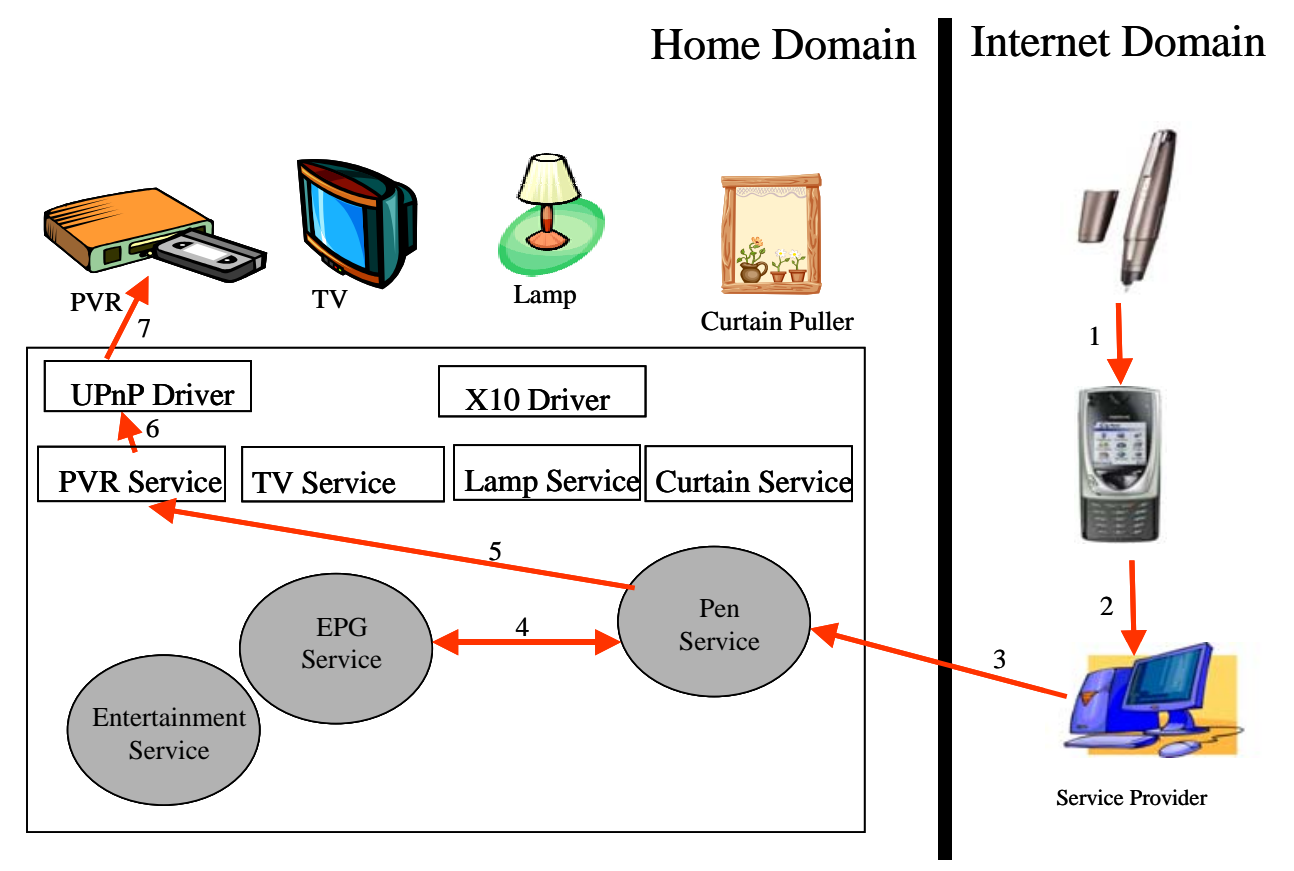

Figure 5: Message Flow for Controlling a PVR with a Digital Pen.

The PVR will confirm the action by a response which is fed back the original path to the pen service and the service provider application. This will generate a SMS message which is sent to the mobile phone originally used to forward the request. This way, the user will receive feedback on the success of the request.

This approach allows a user to program the PVR in his home from anywhere in the world! This approach is very user friendly as using the pen and paper interface fully hides the 
computation and processing of information by computers from the user. As far as the user is concerned no computer use is involved and all they need to do is to tick the right box in the TV magazine.

\subsection{Demo Application}

The approach presented above has been implemented as a practical demonstration (shown at IEEE CCNC 2006 [6]), albeit with a minor change. As no TV magazine was available which supports this technology at the moment, a custom paper form was created which allows users to program their favorite TV shows on their PVRs. The form is shown in Figure 6.

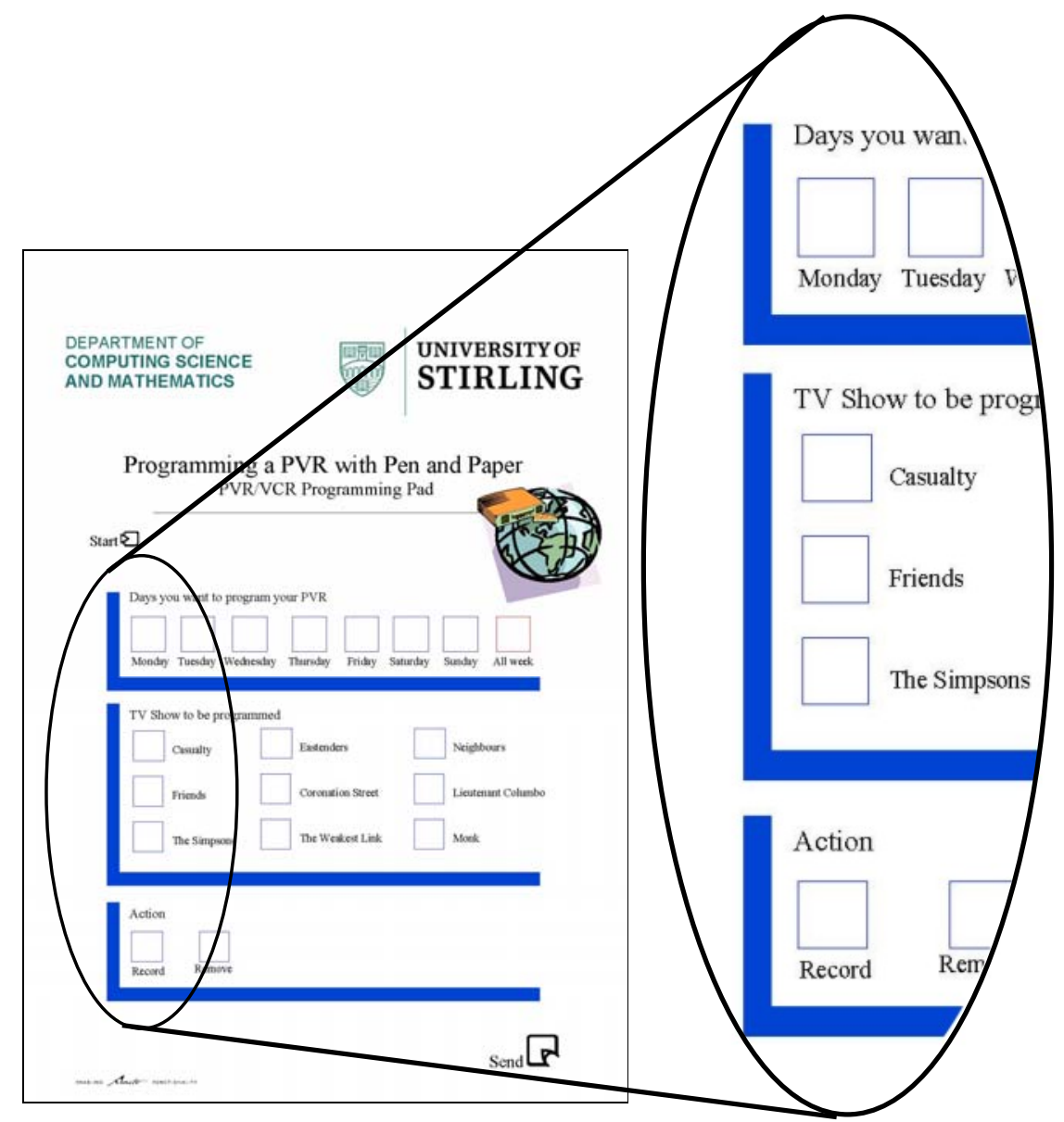

Figure 6: Paper Form to program PVRs.

Here the user ticks the days in the week they want to program the PVR for and the TV show they would like to record. After selecting the required action (Record or Remove) and the Send box, the data is sent to the service provider application and onwards to the user's OSGi gateway as described above. The pen service then looks up the broadcasting time of the selected program on the selected days from the EPG and sends the corresponding requests to the PVR. For the demo, the Knopflerfish OSGi implementation together with a Nebula DigiTV card was used. 
All the pen and paper specific functions needed in the service provider application have been programmed using the libraries and APIs offered by Anoto as part of their Software Development Kit (SDK). The paper has been produced using the Anoto Form Development Kit (FDK). The FDK allows to define custom user areas on the page. Pen strokes within each of these areas can be accessed by the application using API methods offered as part of the SDK. Hence the application can check if there are pen strokes within certain areas on the page. Relating the user areas to the check boxes on the paper form shown in Figure 6, allows to check if a certain box has been ticked. This information can then be easily converted into the XML format as shown above, and forwarded to the user's OSGi gateway.

\subsection{Further Applications of the Approach}

The approach can be extended to other appliances in the home, and indeed beyond the home. Figure 7 shows a paper form to control general aspects of a home [1]. Here the user can specify which appliance in which room is to be controlled.

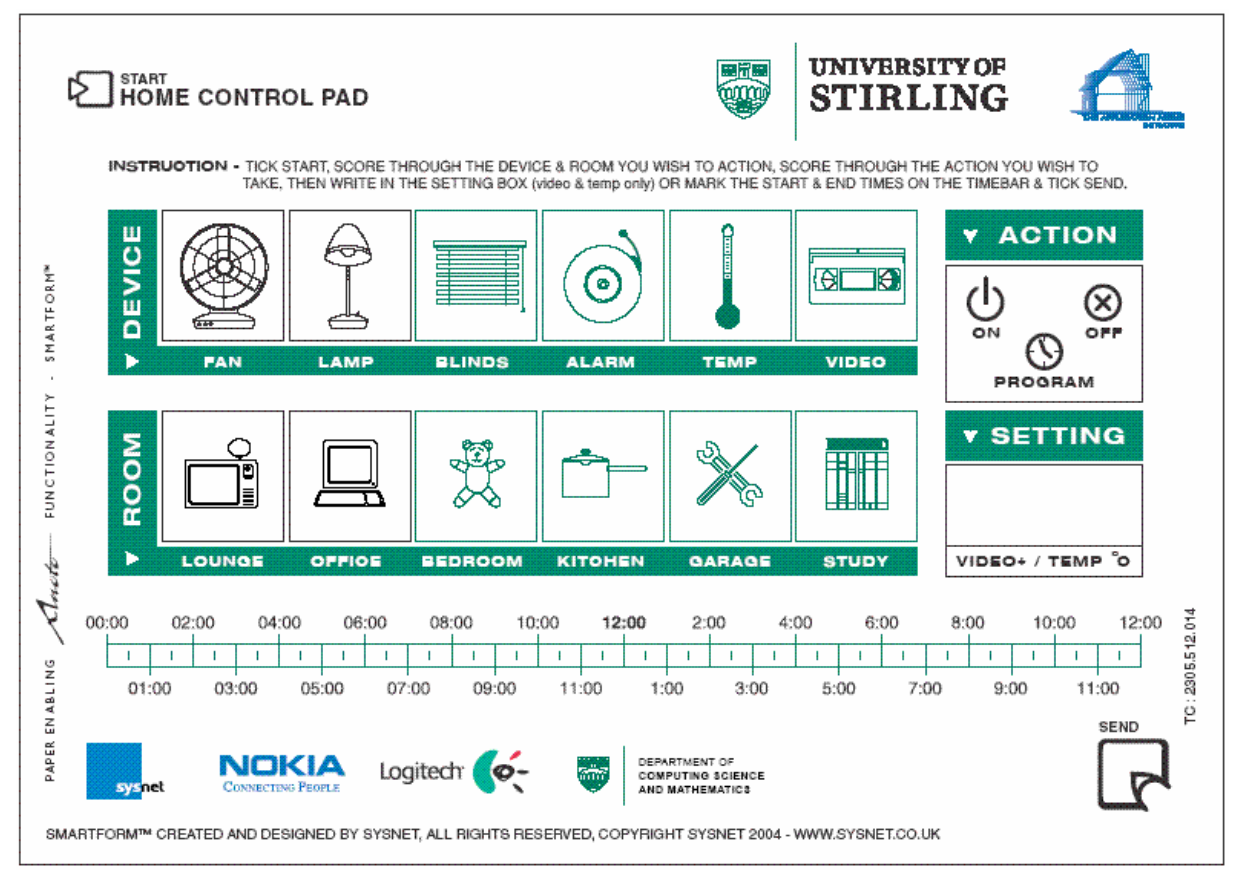

Figure 7: Home Control Pad for controlling general aspects of a home.

This application works essentially in the same way as the one discussed above. The EPG bundle is not required for this application. Instead it uses a bundle which contains information on the location of appliances within the home (e.g. living room lamp). This bundle is interrogated by the pen service for references to appliances matching the location criteria. In a way this bundle extends the OSGi registry which does not hold such information.

A further application of this technology could be within user manuals. Imagine your new TV manual lists all the configuration options as usual, however, you do not need to navigate the complex on-screen menus with the remote control, rather you can directly select an option by ticking the corresponding box in the manual. 
There may also be justification to equip engineers installing for instance heating boilers with a digital pen. To configure the boiler according to the customer's requirements would only take a few ticks with the pen. This would reduce the time the engineer needed to spent at the customer's premises and hence reduce costs.

An application outside the home is the configuration of cars at a car hire station. When a customer hires a car they are presented with a paper form (and a digital pen) where they select their preferences, such as body size, desired temperature, and taste of music. This information is then sent to the car they are about to hire. By the time the customer approaches the car, seats, mirrors, climate control and radio are setup correctly. Implementation of the approach is made easier by the increasing support of OSGi by a number of major car manufacturers [7]. A form for such an application is shown in Figure 8.

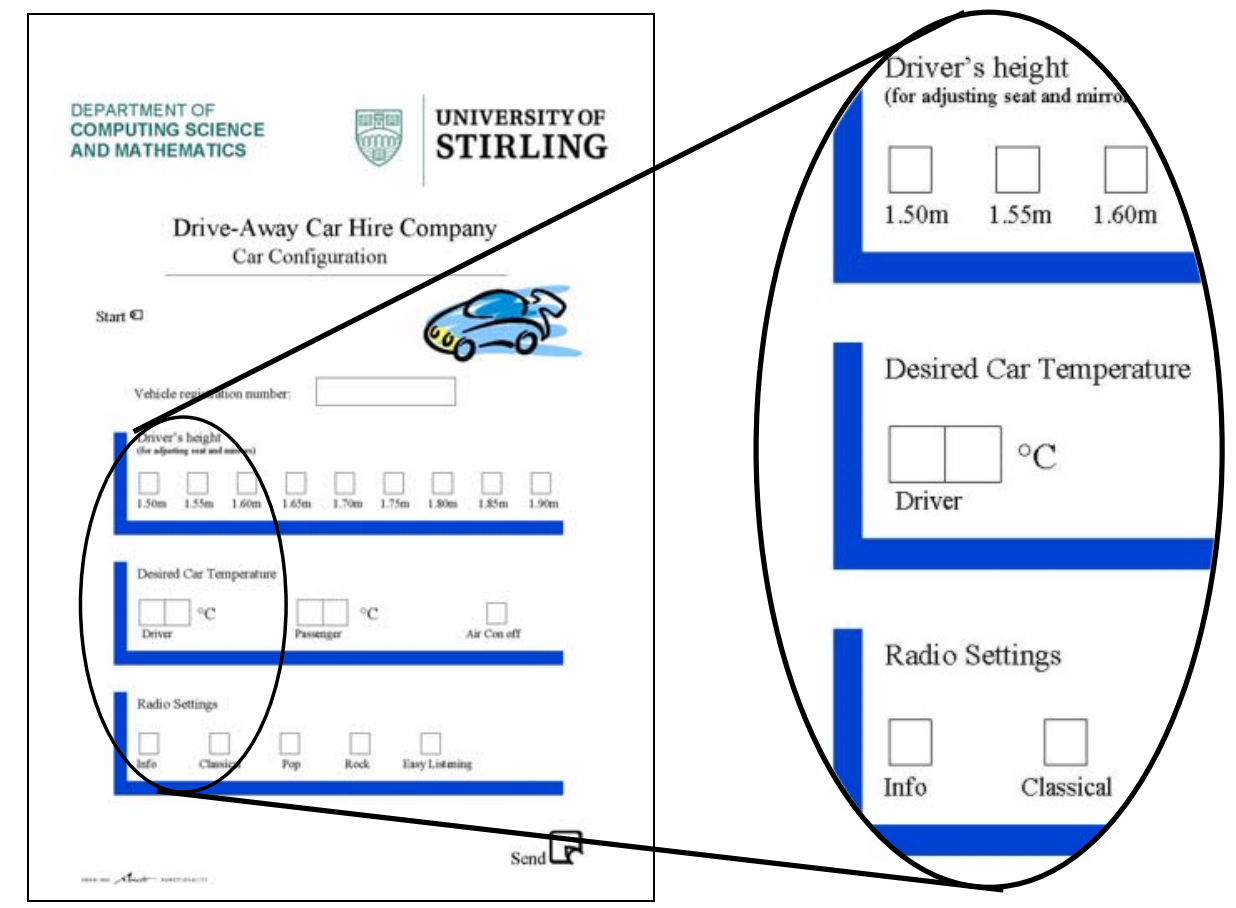

Figure 8: Form for configuration of a car.

\section{Evaluation and Conclusions}

This paper addresses the control of complex networked appliances. Currently, a standard computer interface is most often used to configure and remotely control these appliances. This is frequently unsuitable for the target audience - which is often inexperienced with the use of computers. Furthermore, some users may find it unsuitable to use a laptop or PDA to control their home appliances at certain situations.

This paper argues that Anoto enabled pen and paper are a suitable alternative. Users are highly familiar with pen and paper. An important barrier to adoption of this technology is avoided. Using Bluetooth and the mobile telecommunications network, the data is transferred to a service provider where it is processed and sent to the user's home. Here the OSGi 
gateway offers a single point of control to execute the requests to appliances inside the home. Because the approach employs the use of OSGi it is independent of the protocol used to communicate with the appliance. As has been shown, the approach can be used to control a number of different appliances in the home and also outside the home. Furthermore, all required technologies for this approach are readily commercially available.

The presented approach has been implemented and demoed on different continents at various occasions, including at the IEEE Consumer Communications and Networking Conference in 2006 (IEEE CCNC), and audiences, including communications experts, representatives from appliance manufacturers, teachers from local schools, and students. The feedback has been consistently positive. Communications experts are often intrigued about the versatility of the approach. A number of them came up with a number of additional applications the approach can be applied to and some major appliance manufacturers have expressed interest. End users like the ease of use of the technology.

Modern networked appliances will support complex functions. The pen and paper technology fully hides this complexity and any PC based processing of information from the user. The technology works reliably and is very flexible in terms of the possible applications as is shown by the different examples presented in this paper.

\section{References}

[1] M. Kolberg, E.H. Magill, M. Wilson, P. Burtwistle, and O. Ohlstenius. Controlling Appliances with Pen and Paper, $2^{\text {nd }}$ IEEE Consumer Communications and Networking Conference (CCNC) 2005.

[2] P. Dobrev, D. Famolari, C. Kurzke, and B.A. Miller, Device and service discovery in home networks with OSGi, IEEE Communications Magazine, vol. 40, no. 8, Aug 2002 pp. 86-92.

[3] Open Services Gateway Initiative, OSGi Service Platform Release 3, IOS Press, 2003.

[4] D. Marples and P. Kriens, The Open Services Gateway initiative: An introductory overview, IEEE Communications Magazine, vol. 39, no. 12, December 2001 pp. 110-114.

[5] A. Brown, M. Kolberg, D. Bushmitch, G. Lomako, and M. Ma, A SIP-based OSGi Device Communication Service for Mobile Personal Area Networks, $3^{\text {rd }}$ IEEE Consumer Communications and Networking Conference (CCNC) 2006, Las Vegas, USA.

[6] M. Kolberg and E.H. Magill, Programming a PVR with Pen and Paper, Demo at $3^{\text {rd }}$ IEEE Consumer Communications and Networking Conference (CCNC) 2006, Las Vegas, USA.

[7] GST Forum, Global System for Telematics, EU IST Project, http://www.gstforum.org. 